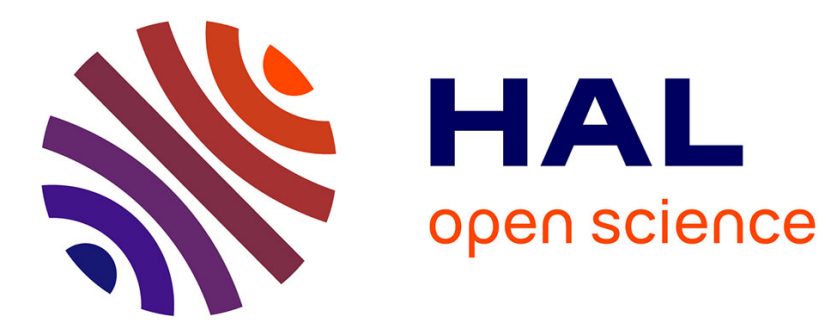

\title{
Universal Long Ranged Correlations in Driven Binary Mixtures
}

Alexis Poncet, Olivier Bénichou, Vincent Démery, Gleb Oshanin

\section{To cite this version:}

Alexis Poncet, Olivier Bénichou, Vincent Démery, Gleb Oshanin. Universal Long Ranged Correlations in Driven Binary Mixtures. Physical Review Letters, 2017, 118 (11), 10.1103/PhysRevLett.118.118002 . hal-02323275

\section{HAL Id: hal-02323275 \\ https://hal.science/hal-02323275}

Submitted on 21 Oct 2019

HAL is a multi-disciplinary open access archive for the deposit and dissemination of scientific research documents, whether they are published or not. The documents may come from teaching and research institutions in France or abroad, or from public or private research centers.
L'archive ouverte pluridisciplinaire HAL, est destinée au dépôt et à la diffusion de documents scientifiques de niveau recherche, publiés ou non, émanant des établissements d'enseignement et de recherche français ou étrangers, des laboratoires publics ou privés. 


\title{
Universal Long Ranged Correlations in Driven Binary Mixtures
}

\author{
Alexis Poncet, ${ }^{1,2}$ Olivier Bénichou, ${ }^{3}$ Vincent Démery, ${ }^{1}$ and Gleb Oshanin ${ }^{3}$ \\ ${ }^{1}$ Gulliver, CNRS, ESPCI Paris, PSL Research University, 10 rue Vauquelin, 75005 Paris, France \\ ${ }^{2}$ Département de Physique, ENS, PSL Research University, 24 Rue Lhomond, 75005 Paris, France \\ ${ }^{3}$ Laboratoire de Physique Théorique de la Matière Condensée, CNRS/UPMC, 4 Place Jussieu, F-75005 Paris, France \\ (Received 6 September 2016; revised manuscript received 6 January 2017; published 15 March 2017)
}

\begin{abstract}
When two populations of "particles" move in opposite directions, like oppositely charged colloids under an electric field or intersecting flows of pedestrians, they can move collectively, forming lanes along their direction of motion. The nature of this "laning transition" is still being debated and, in particular, the pair correlation functions, which are the key observables to quantify this phenomenon, have not been characterized yet. Here, we determine the correlations using an analytical approach based on a linearization of the stochastic equations for the density fields, which is valid for dense systems of soft particles. We find that the correlations decay algebraically along the direction of motion, and have a self-similar exponential profile in the transverse direction. Brownian dynamics simulations confirm our theoretical predictions and show that they also hold beyond the validity range of our analytical approach, pointing to a universal behavior.
\end{abstract}

DOI: 10.1103/PhysRevLett.118.118002

Binary driven mixtures, in which two populations of particles are driven in opposite directions, encompass a variety of systems, ranging from pedestrian traffic [1-3] to charged particle systems like colloidal suspensions [4], electrolytes [5,6], plasmas [7], or ionic liquids [8]. For a large enough driving force, such systems undergo an outof-equilibrium transition into a state in which the particles moving in the same direction spontaneously form lanes, thereby increasing their average velocity $[9,10]$. The nature of the laning transition remains unclear [10-16] and still little is known about the correlations-which are key observables to quantify the transition-and, in particular, about their decay at large distances [14].

Previous studies suggest that these correlations may be long-ranged. Indeed, when only a small fraction of the particles is biased, the correlation between them and the unbiased particles follows the distribution of the density of particles in the wake of a single intruder moving in a quiescent bath, which is relevant for microrheology [17-20]. This distribution has been computed for different models: Brownian dilute hard spheres [21], dense soft spheres [22], or hard core particles on a lattice [23]. In the last two cases, the density behind the intruder was found to approach its unperturbed value as a power law.

The situation is by far less clear when half of the particles are driven in one direction. In this case, the correlations have only recently been investigated using numerical simulations and a numerical integration of a closure of the $N$-particles Fokker-Planck equation [14]. It was shown that the correlations may decay as a power law, with an exponent between 1 and 2, and it was argued that it could be related to a diverging correlation length, and hence, to a true phase transition. However, this observation seemingly contradicts the existence of a finite correlation length, as measured in numerical simulations of large systems [13]. In view of this apparent controversy, it is desirable to have some analytical predictions on the asymptotic decay of correlations.

Here, we determine the correlations in a model driven binary mixture using an analytical approach based on a linearized stochastic density functional theory (SDFT). This approach was introduced recently to compute the correlations in an electrolyte [6] and is reliable for dense systems of soft particles. We obtain that the correlations decay as a power law with an exponent $(d+1) / 2$ at any value of the external force, in both directions along the field, and have a self-similar exponential profile in the transverse direction. Brownian dynamics simulations of harmonic spheres agree quantitatively well with our predictions, and also demonstrate that the scaling and the shape of the correlations hold beyond the validity range of our theory. This observation suggests a universal character of the behavior of correlations.

Model. - We consider a simple model of a binary driven mixture which comprises $N$ colloidal particles, suspended in a solvent which acts as a heat bath at temperature $T$, and interacting via an isotropic pair potential $V(x)$. A fraction $\tau=\tau_{1}$ of the particles - called the "tracers" in what followsis also subject to an external constant force $\boldsymbol{F}[10,13,14]$ (Fig. 1). We assume an overdamped Langevin dynamics so that the coordinates $\boldsymbol{x}_{i}(t) \in \mathbb{R}^{d}$ of the particles obey

$$
\dot{\boldsymbol{x}}_{i}(t)=\mathbb{1}_{i \in \mathcal{T}_{1}} \boldsymbol{F}-\sum_{j \neq i} \nabla V\left[\boldsymbol{x}_{i}(t)-\boldsymbol{x}_{j}(t)\right]+\boldsymbol{\eta}_{i}(t),
$$

where the Gaussian white noise $\boldsymbol{\eta}_{i}(t)$ has a correlation function $\left\langle\boldsymbol{\eta}_{i}(t) \boldsymbol{\eta}_{j}\left(t^{\prime}\right)^{T}\right\rangle=2 T \delta_{i j} \delta\left(t-t^{\prime}\right) \mathbb{1}$, the mobility is 


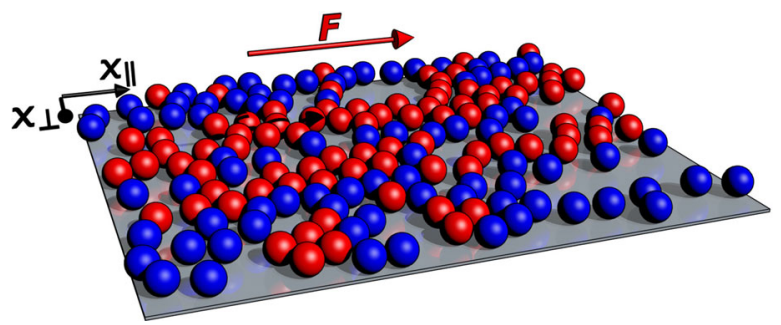

FIG. 1. Snapshot of numerical simulations of Brownian harmonic spheres with $\bar{\rho}=0.5, \tau=0.5, T=10^{-4}$, and $F=0.005$. The tracers (red) are subject to an external force $\boldsymbol{F}$ while the nontracers (blue) are not. Particles of the same species tend to form lanes in the direction of the field.

set to $\kappa=1, \mathcal{T}_{1}$ is set of tracers, and $\mathcal{T}_{2}$ is the set of "nontracers", present at fraction $1-\tau=\tau_{2}$.

This model can also describe situations where the two species are submitted to different external forces, since by symmetry only the difference of the forces applied to the two species affects the correlations [10]. Following Refs. $[10,13,14]$, we do not consider hydrodynamic interactions. This approximation should be valid if the hydrodynamic radius of the particles is smaller than the range of the interaction $V(\boldsymbol{x})$ [24], or if the hydrodynamic interactions are screened [4,25] (e.g., in electrophoresis).

In order to quantify the cooperative effects, we introduce the effective mobility $\kappa_{\text {eff }}$ of the tracers as $\left\langle\dot{\boldsymbol{x}}_{i}\right\rangle=\kappa_{\text {eff }} \boldsymbol{F}$ for $i \in \mathcal{T}_{1}$. In the absence of interactions, the mobility is $\kappa_{\text {eff }}=1$. Using symmetry arguments, one can show that $\kappa_{\text {eff }}(\tau)=1-(1-\tau) K(\tau)$, where $K(\tau)=K(1-\tau)$ [26]. The factor $1-\tau$ comes from the density of nontracers that hinder the motion of the tracers, and $K(\tau)$ describes the cooperativity of the tracers.

The microscopic density of the two species and the pair correlation functions are defined by

$$
\begin{gathered}
\rho_{\alpha}(\boldsymbol{x}, t)=\sum_{i \in \mathcal{T}_{\alpha}} \delta\left(\boldsymbol{x}-\boldsymbol{x}_{i}(t)\right), \\
h_{\alpha \beta}(\boldsymbol{x})=\left\langle\left(\frac{\rho_{\alpha}(\boldsymbol{x})}{\bar{\rho}_{\alpha}}-1\right)\left(\frac{\rho_{\beta}(0)}{\bar{\rho}_{\beta}}-1\right)\right\rangle,
\end{gathered}
$$

where $\alpha=1$ for tracers, 2 for nontracers, and $\bar{\rho}_{\alpha}=\tau_{\alpha} \bar{\rho}=$ $\tau_{\alpha} N / \mathcal{V}, \mathcal{V}$ being the volume of the system. Averaging Eq. (1), we find that $K$ can be expressed via the pair correlation functions as [26]

$$
K=\frac{\bar{\rho}}{F} \int \partial_{\|} V(\boldsymbol{x}) h_{12}(\boldsymbol{x}) d \boldsymbol{x}
$$

the index $\|$ denotes the direction parallel to $\boldsymbol{F}$.

Mean-field calculation.-In order to compute the correlation functions, we use the Dean-Kawasaki equation (or SDFT) for the density fields $[6,27,28]$,

$\dot{\rho}_{\alpha}=\nabla \cdot\left[T \nabla \rho_{\alpha}-\delta_{\alpha 1} \rho_{\alpha} \boldsymbol{F}+\rho_{\alpha} \sum_{\beta} \nabla V * \rho_{\beta}+\rho_{\alpha}^{1 / 2} \boldsymbol{\eta}_{\alpha}\right]$,

where $\boldsymbol{\eta}_{\alpha}(\boldsymbol{x}, t)$ is a Gaussian noise with correlations $\left\langle\boldsymbol{\eta}_{\alpha}(\boldsymbol{x}, t) \boldsymbol{\eta}_{\beta}\left(\boldsymbol{x}^{\prime}, t^{\prime}\right)^{T}\right\rangle=2 T \delta_{\alpha \beta} \delta\left(\boldsymbol{x}-\boldsymbol{x}^{\prime}\right) \delta\left(t-t^{\prime}\right) \mathbb{1}$. This equation is exact, but it is nonlinear and contains multiplicative noise, so that it is difficult to handle it. However, it can be linearized by assuming small density fluctuations, i.e., $\rho_{\alpha}(\boldsymbol{x}, t)-\bar{\rho}_{\alpha}=\sqrt{\bar{\rho}_{\alpha}} \phi_{\alpha}(\boldsymbol{x}, t) \ll \bar{\rho}_{\alpha}$ [22]. Under this assumption, Eq. (5) reads in Fourier space [with convention $\left.\tilde{h}(\boldsymbol{k})=\int h(\boldsymbol{x}) e^{-i \boldsymbol{k} \cdot \boldsymbol{x}} d \boldsymbol{x}\right]$

$$
\begin{gathered}
\partial_{t} \tilde{\phi}_{\alpha}=-k^{2} \sum_{\beta} \tilde{A}_{\alpha \beta} \tilde{\phi}_{\beta}+\tilde{\xi}_{\alpha}, \\
\tilde{A}(\boldsymbol{k})=T\left(\begin{array}{cc}
1+i \frac{f k_{\|}}{k^{2}}+\tau_{1} \tilde{v}(\boldsymbol{k}) & \sqrt{\tau_{1} \tau_{2}} \tilde{v}(\boldsymbol{k}) \\
\sqrt{\tau_{1} \tau_{2}} \tilde{v}(\boldsymbol{k}) & 1+\tau_{2} \tilde{v}(\boldsymbol{k})
\end{array}\right),
\end{gathered}
$$

where $\tilde{\xi}(\boldsymbol{k}, t)$ is the Gaussian noise with correlations $\left\langle\tilde{\xi}_{\alpha}(\boldsymbol{k}, t) \tilde{\xi}_{\beta}\left(\boldsymbol{k}^{\prime}, t^{\prime}\right)\right\rangle=2(2 \pi)^{d} T \delta_{\alpha \beta} \delta\left(\boldsymbol{k}+\boldsymbol{k}^{\prime}\right) \delta\left(t-t^{\prime}\right) k^{2}, \quad f=$ $F / T$ is the Péclet number and $v=\bar{\rho} V / T$ is the rescaled potential. The eigenvalues of $\tilde{A}(\boldsymbol{k})$ have a positive real part; hence, the fluctuations of the density fields are stable. The linearization of Eq. (5) is valid in the large density limit, $\bar{\rho} \rightarrow \infty$, with $v$ kept constant, meaning that the suspension is very dense and soft [22].

The correlation functions of the fields $\phi, C_{\alpha \beta}(\boldsymbol{x})=$ $\left\langle\phi_{\alpha}(\boldsymbol{x}, t) \phi_{\beta}(0, t)\right\rangle$ are related to the pair correlation functions (3) by $h_{\alpha \beta}=C_{\alpha \beta} / \sqrt{\bar{\rho}_{\alpha} \bar{\rho}_{\beta}}$. In the stationary regime, they satisfy [29]

$$
\tilde{A} \tilde{C}+\tilde{C} \tilde{A}^{*}=2 T \mathbb{1} .
$$

At equilibrium, i.e., without external force, $\tilde{A}^{*}=\tilde{A}$, the equilibrium correlation functions $\tilde{C}=T \tilde{A}^{-1}$ are recovered. For an arbitrary force, the solution reads [26]

$$
\tilde{C}=\left[(1+\tilde{v})(2+\tilde{v})^{2}+\left(1+\tilde{v}+\gamma \tilde{v}^{2}\right) \frac{f^{2} k_{\|}^{2}}{k^{4}}\right]^{-1}\left(\begin{array}{cc}
\left(1+\tau_{2} \tilde{v}\right)\left[(2+\tilde{v})^{2}+\frac{f^{2} k_{\|}^{2}}{k^{4}}\right] & -\sqrt{\gamma} \tilde{v}(2+\tilde{v})\left(2+\tilde{v}-i \frac{f k_{\|}}{k^{2}}\right) \\
-\sqrt{\gamma} \tilde{v}(2+\tilde{v})\left(2+\tilde{v}+i \frac{f k_{\|}}{k^{2}}\right) & \left(1+\tau_{1} \tilde{v}\right)\left[(2+\tilde{v})^{2}+\frac{f^{2} k_{\|}^{2}}{k^{4}}\right]
\end{array}\right),
$$



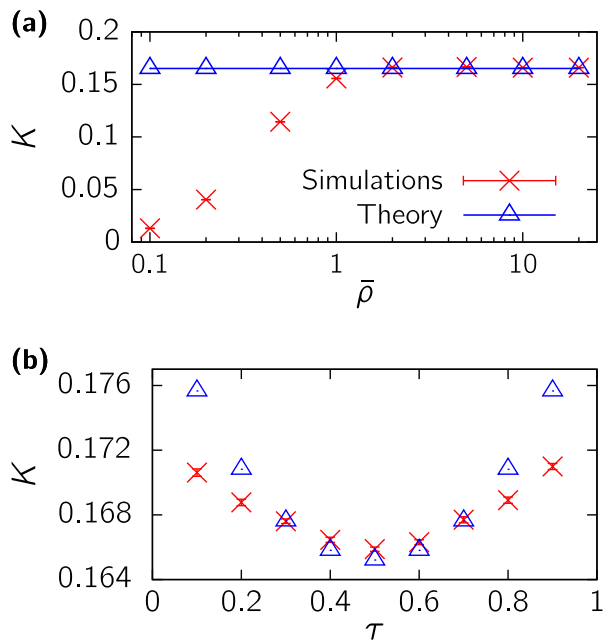

FIG. 2. Cooperativity $K$ in $d=2$ as a function (a) of $\bar{\rho}$ with $\bar{\rho} / T=10, F / T=20$, and $\tau=0.5$, (b) of the fraction of tracers $\tau$ for $\bar{\rho}=2, \bar{\rho} / T=10$, and $F / T=20$. Solid lines and points denote analytical and simulation results, respectively.

where $\gamma=\tau(1-\tau)$. Equation (9) allows us to determine the function $K$ entering the mobility:

$K=\frac{1}{\bar{\rho}} \int \frac{\tilde{v}^{2}(2+\tilde{v}) k_{\|}^{2} k^{2}}{(1+\tilde{v})(2+\tilde{v})^{2} k^{4}+\left[1+\tilde{v}+\gamma \tilde{v}^{2}\right] f^{2} k_{\|}^{2}} \frac{d \boldsymbol{k}}{(2 \pi)^{d}}$.

In the limit $\tau=0$, the mobility found for a single tracer is recovered [Eq. (79) of Ref. [22]]. $K$ is minimal (i.e., the cooperativity is maximal) for $\tau=1 / 2$ (Fig. 2), consistently with the symmetry $K(\tau)=K(1-\tau)$ and the absence of cooperativity in the limit $\tau \rightarrow 0$.

Large scale behavior.-The Fourier transforms of the correlation functions Eq. (9) are not continuous at 0. The long distance behavior in real space can be obtained by keeping only their singular part [26], leading to

$$
h_{\alpha \beta}(\boldsymbol{x}) \approx_{x_{\|} \rightarrow \pm \infty} \frac{H_{\alpha \beta}^{ \pm}}{\left|x_{\|}\right|^{\frac{d+1}{2}}} g\left(\frac{\boldsymbol{x}_{\perp}}{\sqrt{D\left|x_{\|}\right|}}\right)
$$

where

$$
\begin{gathered}
g(\boldsymbol{u})=\nabla_{\boldsymbol{u}}^{2}\left(e^{-\boldsymbol{u}^{2} / 2}\right)=\left(\boldsymbol{u}^{2}-d+1\right) e^{-\boldsymbol{u}^{2} / 2}, \\
D=\frac{2\left(2+v_{0}\right)}{\beta f}, \quad \beta=\left[1+\gamma \frac{v_{0}^{2}}{1+v_{0}}\right]^{1 / 2}, \\
H_{11}^{ \pm}=-\frac{(1-\tau) v_{0}^{2}\left[1+(1-\tau) v_{0}\right] \beta^{\frac{d-5}{2}} f^{\frac{d-1}{2}}}{2^{d+1} \pi^{\frac{d-1}{2}} \bar{\rho}\left(1+v_{0}\right)^{2}\left(2+v_{0}\right)^{\frac{d-1}{2}}} \\
H_{21}^{ \pm}=\frac{v_{0} \beta^{\frac{d-1}{2}}\left(1 \mp \beta^{-1}\right) f^{\frac{d-1}{2}}}{2^{d+1} \pi^{\frac{d-1}{2}} \bar{\rho}\left(1+v_{0}\right)\left(2+v_{0}\right)^{\frac{d-1}{2}}}
\end{gathered}
$$

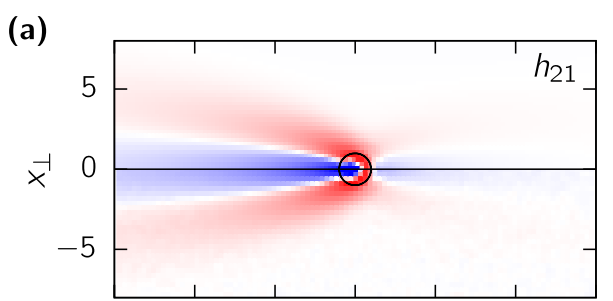

(b)

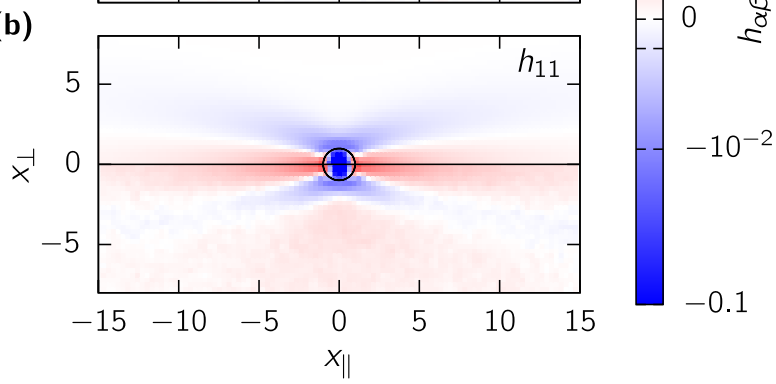

FIG. 3. Pair correlation functions from the simulations (lower half) and the theory (upper half) for $\bar{\rho}=2, T=0.2, F=4$, and $\tau=0.5$, in dimension $d=2$. The black circle sets the size of a particle. (a) Tracer-nontracer correlation. (b) tracer-tracer correlation.

Eqs. (11)-(15) are our main results. First, they not only yield the algebraic decay with power $(d+1) / 2$ of the depleted wake of a single tracer [22], but also generalize this result by defining the shape of the wake. At a vanishing
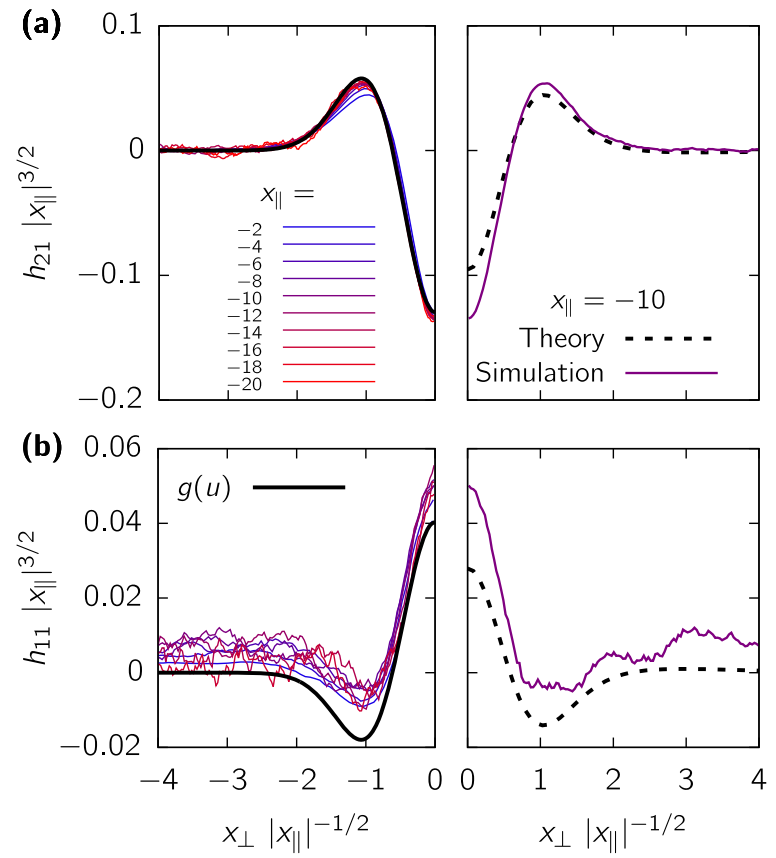

FIG. 4. Rescaled tranverse profiles of the pair correlation functions for $\bar{\rho}=2, T=0.2, F=4, \tau=0.5$, in dimension $d=2$. Left panel: profiles from the simulations at different longitudinal positions and universal shape Eq. (12) (thick black line). Right panel: profiles at $x_{\|}=-10$ from the simulations (solid line) and the analytical prediction obtained by numerical inversion of Eq. (9) (dashed line). (a) Tracer-nontracer correlation. (b) Tracer-tracer correlation. 


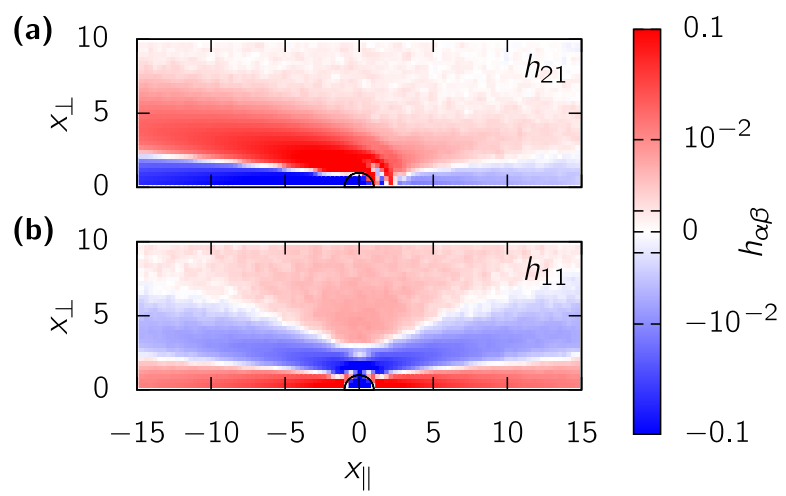

FIG. 5. Pair correlation functions from the simulations for $\bar{\rho}=0.2, T=0.001, F=0.02, \tau=0.5$, in dimension $d=2$. The black circle sets the size of a particle. (a) Tracer-nontracer correlation. (b) Tracer-tracer correlation.

tracers density $(\tau \rightarrow 0), \beta \rightarrow 1$ and $H_{21}^{+} \rightarrow 0$, the decay of $h_{21}$ in front of the tracers is exponential [26]. Second, they show that for a finite density of tracers the algebraic decay holds also for the tracer-nontracer correlation function $h_{21}$ in front of the tracers $\left(x_{\|} \rightarrow \infty\right)$, and for the tracer-tracer correlation function $h_{11}$. The tracer-tracer correlation is positive along the axis $\boldsymbol{x}_{\perp}=0$ [Fig. 3(b)], indicating a propensity for forming lanes.

Numerical simulations. - We have performed two dimensional Brownian dynamics simulations of Eq. (1) with $N=$ $8 \times 10^{4}$ harmonic spheres $\left[V(\boldsymbol{x})=(1-|\boldsymbol{x}|)^{2} \theta(1-|\boldsymbol{x}|) / 2\right.$, where $\theta(u)$ is the Heaviside function], in a square box with periodic boundary conditions [26,30].

In Fig. 2(a) we show the cooperativity as a function of $\bar{\rho}$ with $\bar{\rho} / T$ and $f$ kept constant. We observe that the simulation results converge to the analytical prediction as $\bar{\rho} \rightarrow \infty$, which is the condition of validity of our computation. The cooperativity displays a minimum at $\tau=0.5$, which is correctly predicted by the theory [see Fig. 2(b)].

The correlation functions for $\bar{\rho}=2, T=0.2$, and $f=20$ are shown in Fig. 3, and are compared to the numerical integration of Eq. (9). Cuts along the transverse direction, rescaled using Eq. (11), are compared to the numerical integration and to the shape Eq. (12) in Fig. 4. A very good collapse is found, which confirms the scaling laws, and a quantitative agreement is found for the shape.

Universality.-We now show that the scaling (11) of the correlations and their shape (12), hold far beyond the validity regime of our computation, namely, a dense and soft suspension. We simulated our system of harmonic spheres in the dilute and hard regime: $\bar{\rho}=0.2, T=10^{-3}$, $f=20$. The correlations are shown in Fig. 5; they have the same shape as in the opposite limit of dense and soft particles. Rescaled transverse cuts are shown on Fig. 6; a very good collapse is found, and the shape is well described by Eq. (12) with fitted height and width.

These results suggest that the long-range behavior of the correlations, Eqs. (11), (12), is universal. It has a simple
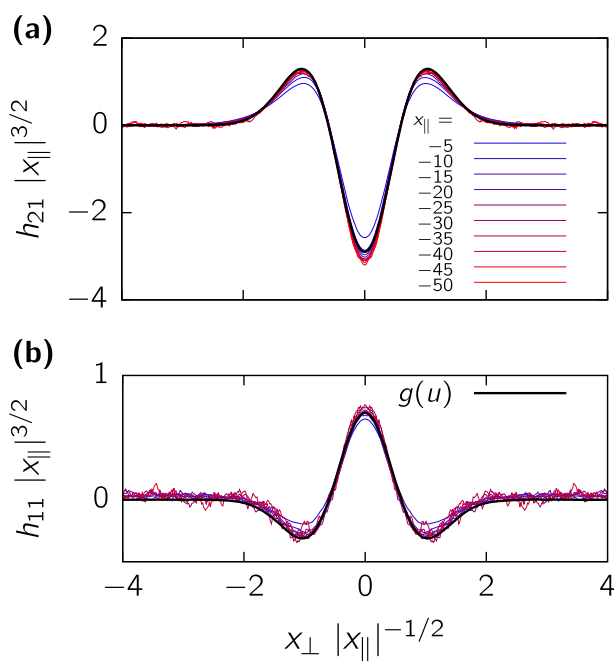

FIG. 6. Rescaled tranverse profiles of the pair correlation functions for $\bar{\rho}=0.2, T=0.001, F=0.02, \tau=0.5$, in dimension $d=2$. The transverse profiles from the simulations at different longitudinal positions are shown together with the universal shape Eq. (12) with fitted height and width (thick black line). (a) Tracer-nontracers correlation. (b) Tracer-tracer correlation.

interpretation as a diffusive process. Replacing $x_{\|}$(or $-x_{\|}$, depending on the direction of interest) by the time and $\boldsymbol{x}_{\perp}$ by the position, Eq. (11) is the solution of a diffusion equation where the source is the second derivative of a Dirac distribution (notably, it is symmetric and its integral is zero). This behavior emanates from simple properties of our system: (i) The long-distance dynamics without external force is diffusive [i.e., the relaxation rate of $\tilde{\phi}(\boldsymbol{k})$ scales as $k^{2}$ for small $k$ ]; (ii) the tracers have a finite average velocity; (iii) the number of particles is conserved. We conclude that the large scale behavior of the correlations is universal and should be observed in the systems sharing these properties.

Discussion.-We have shown that the correlation functions in a simple model of a driven binary mixture decay algebraically in the direction of the external force, and have a self-similar profile in the direction perpendicular to the external force. This shape is universal and should hold in any system which is diffusive at large scales and where the external field induces a finite average velocity of the particles.

Our results shed light on the previous observations. First, we confirm the conjecture of Ref. [14]: the correlation functions are long-ranged with an exponent $3 / 2$ in $d=2$, which is compatible with the value reported in Ref. [14]. However, our conclusion is different: (i) this algebraic decay is not associated with the divergence in the structure factors, (ii) it does not indicate a phase transition, instead it is observed over a whole region of the parameter space. Second, in agreement with Ref. [13], we find no trace of a phase transition. However, we show that the correlation length defined in Ref. [13] does not correspond to an exponential 
decay of the correlations, as is usually the case; resolving this apparent contradiction certainly deserves future work.

In the model considered here, the system is fluid, the dynamics is overdamped and the interactions are shortranged. However, driven binary mixtures may be very different: they may have an underdamped dynamics $[7,31,32]$ (e.g., in plasmas), or even an active dynamics [3], long-ranged interactions (electrostatic [6] or hydrodynamic), a solidlike behavior in the absence of an external force (e.g., if the system is glassy) [33], etc. It seems natural to inquire next how these differences would affect the large distance behavior of the correlation functions, and to relate it with the nature of the laning transition in these systems.

The authors thank D. S. Dean for stimulating discussions. The work of O. B. is supported by the European Research Council (Grant No. FPTOpt-277998).

[1] M. Moussaïd, E. G. Guillot, M. Moreau, J. Fehrenbach, O. Chabiron, S. Lemercier, J. Pettré, C. Appert-Rolland, P. Degond, and G. Theraulaz, Traffic instabilities in selforganized pedestrian crowds, PLoS Comput. Biol. 8, e1002442 (2012).

[2] J. Cividini, C. Appert-Rolland, and H. J. Hilhorst, Diagonal patterns and chevron effect in intersecting traffic flows, Europhys. Lett. 102, 20002 (2013).

[3] N. Bain and D. Bartolo, Critical mingling and universal correlations in binary active liquids, arXiv:1612.02565.

[4] T. Vissers, A. Wysocki, M. Rex, H. Lowen, C. Patrick Royall, A. Imhof, and A. van Blaaderen, Lane formation in driven mixtures of oppositely charged colloids, Soft Matter 7, 2352 (2011).

[5] R. R. Netz, Conduction and diffusion in two-dimensional electrolytes, Europhys. Lett. 63, 616 (2003).

[6] V. Démery and D.S. Dean, The conductivity of strong electrolytes from stochastic density functional theory, J. Stat. Mech. (2016) P023106.

[7] K. R. Sütterlin, A. Wysocki, A. V. Ivlev, C. Räth, H. M. Thomas, M. Rubin-Zuzic, W. J. Goedheer, V. E. Fortov, A. M. Lipaev, V. I. Molotkov, O. F. Petrov, G. E. Morfill, and H. Löwen, Dynamics of Lane Formation in Driven Binary Complex Plasmas, Phys. Rev. Lett. 102, 085003 (2009).

[8] A. A. Lee, S. Kondrat, G. Oshanin, and A. A. Kornyshev, Charging dynamics of supercapacitors with narrow cylindrical nanopores, Nanotechnology 25, 315401 (2014).

[9] D. Helbing, I. J. Farkas, and T. Vicsek, Freezing by Heating in a Driven Mesoscopic System, Phys. Rev. Lett. 84, 1240 (2000).

[10] J. Dzubiella, G. P. Hoffmann, and H. Löwen, Lane formation in colloidal mixtures driven by an external field, Phys. Rev. E 65, 021402 (2002).

[11] J. Chakrabarti, J. Dzubiella, and H. Löwen, Dynamical instability in driven colloids, Europhys. Lett. 61, 415 (2003).

[12] J. Chakrabarti, J. Dzubiella, and H. Löwen, Reentrance effect in the lane formation of driven colloids, Phys. Rev. E 70, 012401 (2004).

[13] T. Glanz and H. Löwen, The nature of the laning transition in two dimensions, J. Phys. Condens. Matter 24, 464114 (2012).
[14] M. Kohl, A. V. Ivlev, P. Brandt, G. E. Morfill, and H. Löwen, Microscopic theory for anisotropic pair correlations in driven binary mixtures, J. Phys. Condens. Matter 24, 464115 (2012).

[15] K. Klymko, P. L. Geissler, and S. Whitelam, Microscopic origin and macroscopic implications of lane formation in mixtures of oppositely driven particles, Phys. Rev. E 94, 022608 (2016).

[16] E. M. Foulaadvand and B. Aghaee, Driven binary colloidal mixture in a 2D narrow channel with hard walls, Eur. Phys. J. E 39, 37 (2016).

[17] A. Meyer, A. Marshall, B. G. Bush, and E. M. Furst, Laser tweezer microrheology of a colloidal suspension, J. Rheol. 50, 77 (2006).

[18] R. Candelier and O. Dauchot, Journey of an intruder through the fluidization and jamming transitions of a dense granular media, Phys. Rev. E 81, 011304 (2010).

[19] L. G. Wilson and W. C. K. Poon, Small-world rheology: an introduction to probe-based active microrheology, Phys. Chem. Chem. Phys. 13, 10617 (2011).

[20] E. Nazockdast and J. F. Morris, Active microrheology of colloidal suspensions: Simulation and microstructural theory, J. Rheol. 60, 733 (2016).

[21] T. M. Squires and J. F. Brady, A simple paradigm for active and nonlinear microrheology, Phys. Fluids 17, 073101 (2005).

[22] V. Démery, O. Bénichou, and H. Jacquin, Generalized Langevin equations for a driven tracer in dense soft colloids: construction and applications, New J. Phys. 16, 053032 (2014).

[23] O. Bénichou, A. M. Cazabat, J. De Coninck, M. Moreau, and G. Oshanin, Stokes Formula and Density Perturbances for Driven Tracer Diffusion in an Adsorbed Monolayer, Phys. Rev. Lett. 84, 511 (2000).

[24] A. S. Khair and J. F. Brady, Single particle motion in colloidal dispersions: A simple model for active and nonlinear microrheology, J. Fluid Mech. 557, 73 (2006).

[25] D. Long and A. Ajdari, A note on the screening of hydrodynamic interactions, in electrophoresis, and in porous media, Eur. Phys. J. E 4, 29 (2001).

[26] See Supplemental Material at http://link.aps.org/ supplemental/10.1103/PhysRevLett.118.118002 for detailed calculations.

[27] K. Kawasaki, Stochastic model of slow dynamics in supercooled liquids and dense colloidal suspensions, Physica (Amsterdam) 208A, 35 (1994).

[28] D. S. Dean, Langevin equation for the density of a system of interacting Langevin processes, J. Phys. A 29, L613 (1996).

[29] C. Gardiner, Stochastic Methods (Springer, Berlin, 2009).

[30] A. Poncet, Zenodo 229897 (2017), https://github.com/ stykoo/BrownianTracers/tree/v1.0.

[31] X. Liu, W. Ge, and J. Li, Non-equilibrium phase transitions in suspensions of oppositely driven inertial particles, Powder Technol. 184, 224 (2008).

[32] V. Démery, Mean-field microrheology of a very soft colloidal suspension: Inertia induces shear thickening, Phys. Rev. E 91, 062301 (2015).

[33] I. Gazuz, A. M. Puertas, Th. Voigtmann, and M. Fuchs, Active and Nonlinear Microrheology in Dense Colloidal Suspensions, Phys. Rev. Lett. 102, 248302 (2009). 